In addition to the peptide mapping studies, we found that the chromobindins were denatured and precipitated by heating to $100{ }^{\circ} \mathrm{C}$ for $10 \mathrm{~min}$, a treatment we used to purify the particularly heat-stable light chains. Therefore, in addition to significant primary structural differences, the light chains have distinct physical chemical properties from the chromobindins. We conclude that adrenal medullary clathrin light chains have not been obtained in the in vitro affinity chromatography experiments.

Calmodulin is one of the chromobindin proteins that can be isolated by $\mathrm{Ca}^{2+}$-dependent affinity chromatography on chromaffin granule membranes ${ }^{2}$. The hypothesis that calmodulin regulates the interaction of the other chromobindins with the membrane is supported by the observation that some of the chromobindins bind in a $\mathrm{Ca}^{2+}$-dependent manner to a calmodulin-Sepharose column ${ }^{5}$. As a more stringent test, we conducted affinity chromatography experiments with adrenal or liver cytosol on chromaffin granule membrane affinity columns in the presence of $100 \mu \mathrm{M}$ trifluoperazine to antagonize calmodulin action. This drug blocked the binding of calmodulin to the affinity columns, but did not significantly alter the spectrum of other chromobindins obtained (Fig. 2). Therefore, calmodulin does not appear to be required for the recruitment of these other proteins to the membrane. The binding of some of these proteins to a calmodulin-Sepharose column may have been due to their ability to interact in a $\mathrm{Ca}^{2+}$-dependent manner with a hydrophobic surface, either on the calmodulin molecule, or on membrane lipid. The presence of calmodulin in the chromobindin class of proteins may reflect direct interaction with mem- brane proteins, or an indirect interaction through the other chromobindins that does not influence the membrane binding properties of these proteins. Thus, with the exception of synexin which may initiate intermembrane contact and fusion ${ }^{10-12}$, it is unclear what the roles of the membrane binding proteins we have characterized may be in the secretory pathway.

We thank James Keen for providing a sample of bovine tropomyosin, Virginia Simnad for help in the trifluoperazine experiments and Lisa Dowling for technical assistance. This study was supported by the NSF (PCM-82-06453), the Virginia Affiliate of the American Heart Association, the Council for Tobacco Research and the University of Virginia Diabetes Research and Training Center.

Received 13 October; accepted 5 December 1983

1. Creutz, C. E. Biochem. biophys. Res. Commun. 103, 1395-1400 (1981)

2. Creutz, C. E. et al. J. biol. Chem. 258, 14664-14674 (1983)

3. Geisow, M. J. \& Burgoyne, R. D. J. Neurochem. 38, 1735-1741 (1982).

4. Brocklehurst, K. W. \& Hutton, J. C. Biochem. J. 210, 533-539 (1983).

5. Geisow, M. J. \& Burgoyne, R. D. Nature 301, 432-435 (1983).

6. Blaschko, H., Combine, R. S., Schneider, F. H., Silver, M. \& Smith, A. D. Nature 215, 58-59 (1967).

7. Winkler, H. Phil. Trans. R. Soc. B261, 293-303 (1971).

8. Elder, J. H., Pickett, R. A., Hampton, J. \& Lerner, R. A. J. biol. Chem. 252, 6510-6515 (1977).

9. Keen, J. H., Willingham, M. C. \& Pastan, I. H. Cell 16, 303-312 (1979).

10. Creutz, C. E., Pazoles, C. J. \& Pollard, H. B. J. biol. Chem, 253, 2858-2866 (1978)

11. Creutz, C. E. \& Sterner, D. C. Biochem. biophys. Res. Commun. 114, 355-364 (1983).

12. Creutz, C. E. J. Cell Biol. 91, 247-256 (1981).

H13. Brodsky, F. M., Holmes, N. J. \& Parham, P. J. Cell Biol. 96, 911-914 (1983).

14. Laemmli, U. K. Nature 227, 680-685 (1970).

\section{A model for the cosmic creation of nuclear energy - a reassessment}

\section{K.-E. Eriksson, S. Islam \& B.-S. Skagerstam}

Nature 296, 540-543 (1982)

THIS letter included an unfortunate mistake which affects the loss of exergy in helium synthesis and hence the total exergy per nucleon. Naively one would expect the exergy per nucleon to be $b_{4}$ (the binding energy per nucleon in ${ }^{56} \mathrm{Fe}$ ) apart from the loss due to helium formation. With a fraction $\gamma_{0}$ of neutrons immediately before the helium synthesis, this loss could be expected to be $2 \gamma_{0} b_{3}$ where $b_{3}$ is the ${ }^{4} \mathrm{He}$ binding energy per nucleon. Thus for a cooling universe $(T \rightarrow 0)$, the exergy per nucleon $E(T)$ would approach

$$
E(0)=b_{4}-2 \gamma_{0} b_{3}=6.36 \mathrm{MeV}
$$

instead of the result (11) given in our paper. The simple estimate $\left(11^{*}\right)$ turns out to be the right one.

Consider a mixture of four ideal non-degenerate gases (consisting of ${ }^{1} \mathrm{H},{ }^{1} \mathrm{n},{ }^{4} \mathrm{He}$ and ${ }^{56} \mathrm{Fe}$ corresponding to $\left.i=1,2,3,4\right)$ in a volume $V$. Let $n_{i}$ be the number of nuclei of the $i$ th kind, so that in all there are $N=\sum_{i} n_{i} A_{i}$ nucleons. Since radiation dominates, equilibrium temperature and equilibrium pressure prevail, and the exergy per nucleon is purely 'chemical'.

$$
E=\frac{1}{N} \sum_{i=1}^{4} n_{i}\left(\mu_{i}-\mu_{i 0}\right)=\frac{1}{N} \sum_{i=1}^{4} n_{i} k T \ln \frac{n_{i} / V}{n_{i}^{(0)} / V}
$$

Here $\mu_{i}$ is the chemical potential of nuclide $i$ and $\mu_{i 0}$ is its equilibrium value corresponding to an equilibrium density $n_{i}^{(0)} / V$. With

$$
\left\{\begin{array}{l}
P_{i}=\frac{n_{i} A_{i}}{N} \\
P_{i}^{(0)}=\frac{n_{i}^{(0)} A_{i}}{N}
\end{array}\right.
$$

the exergy may then be written

$$
E=\sum_{i=1}^{4} P_{i} \frac{k T}{A_{i}} \ln \frac{P_{i}}{P_{i}^{(0)}}
$$

This equation differs from the equation (2) in the paper in that for each nuclide, $T / A_{i}$ apears as an 'effective temperature', taking into account the reduced number of degrees of freedom of bound nucleons.

From the correct equation $\left(2^{*}\right)$ we obtain the correct expression for the exergy per nucleon after helium formation,

$$
E(T)=\mu(T)-2 \gamma_{0} b_{3}-k T \ln \frac{2\left[\kappa r\left(\frac{m c^{2}}{k T}\right)^{3 / 2}\right]^{1-\frac{3}{2} \gamma_{0}}}{\left(1-2 \gamma_{0}\right)^{1-2 \gamma_{0}} \gamma_{0}^{\frac{1}{2} \gamma_{0}}}
$$

with $\mu(T)$ approaching $b_{4}$ for small $T$ then $\left(11^{*}\right)$ obtains.

Figure 2 of the paper has to be modified, and the corrected Fig. 2 (below) shows the creation of exergy as given by (9) and $\left(10^{*}\right)$.

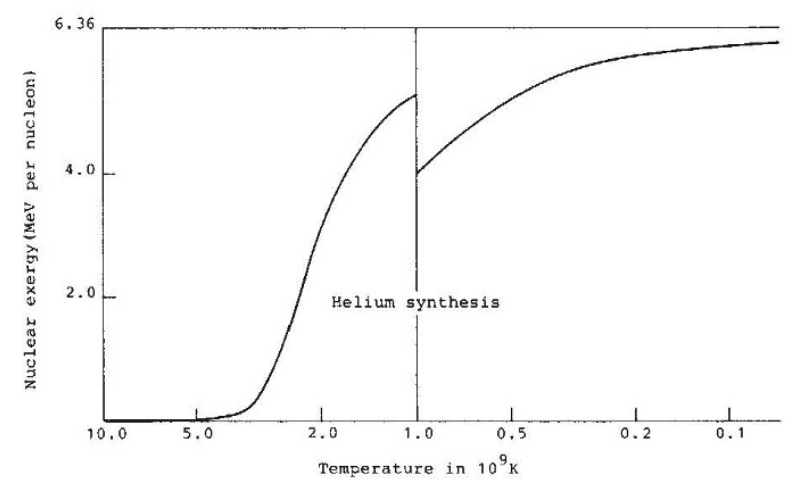

A couple of important references should also be added. Davis' book ${ }^{1}$ on time asymmetry should have been referred to in the original paper. Frautschi's later paper ${ }^{2}$ on negentropy in the Universe is also relevant. The model has been discussed by van $\mathrm{Hove}^{3}$ in connection to other time-asymmetric processes of the early Universe.

\footnotetext{
1. Davies, P. C. W. The Physics of Time Asymmetry (University of California Press, Berkeley, 1974)

2. Frautschi, S. Science 217, 593 (1982)

3. Van Hove, L. Non-equilibrium Processes in Early Cosmology (Preprint no TH.3623-CERN,
} Geneva, 1983) 